\title{
Preparation and Evaluation of Sulfasalazine Nanoparticles by Desolvation Technique
}

Amand Alekhya ${ }^{1}$, Abbaraju Krishna Sailaja ${ }^{1^{*}}$

${ }^{1}$ Department of Pharmaceutics, RBVRR Women's College of Pharmacy. Affiliated to Osmania University, Hyderabad, India.

*Corresponding author: A. Krishna Sailaja, Department of Pharmaceutics, RBVRR Women's College of Pharmacy, Affiliated to Osmania University, Hyderabad, India.

Received date: April 01, 2021; Accepted date: April 21, 2021; Published date: April 30,2021

Citation: Amand Alekhya, A. Krishna Sailaja, (2021). Preparation and Evaluation of Sulfasalazine Nanoparticles by Desolvation Technique. $J$ Clinical Research and Reports, 7(5); DOI:10.31579/2690-1919/160

Copyright: (C) 2021, A. Krishna Sailaja. This is an open access article distributed under the Creative Commons Attribution License, which permits unrestricted use, distribution, and reproduction in any medium, provided the original work is properly cited.

\begin{abstract}
Sulfasalazine is a combination of an aspirin like anti-inflammatory component and a sulfa antibiotic like component. It is an Anti-inflammatory agent used to relive the pain of the Bowel diseases, Rheumatoid arthritis, Psoriatic arthritis, Crohn's disease and Ulcerative colitis. Sulfasalazine acts to deplete the damage to the joints, rather than just relieve the pain, it belongs to the group of medicines called disease modifying anti-rheumatic drugs (DMARDs).

Methodology: The objective of the present study is to prepare, optimize the nanoparticles containing sulfasalazine and bovine serum albumin (BSA) by using desolvation method, and to study the effect of process variables like stirring speed on product yield, drug content. Glutaraldehyde is used as crosslinking agent and sulfasalazine is used as model drug.

Results: The prepared nanoparticles showed maximum drug content, for continuous method-97.27\% and for intermittent method-95.08\%. The prepared nanoparticles showed good product yield of $96.26 \%$ (intermittent method) and $98.4 \%$ (continuous method). Invitro release of sulfasalazine loaded nanoparticles showed good sustained release and maximum drug release of $38.05 \%$ for continuous method and $40.73 \%$ for intermittent method within 6 hours interval.
\end{abstract}

Keywords: sulfasalazine; anti-inflammatory component; sulfa antibiotic; bovine serum albumin (BSA); glutaraldehyde; desolvation technique; nanoparticles; DMARDs; DMSO

\section{Introduction:}

1.1 Definition: Nanoparticles are solid colloidal particles ranging from 1 to $1000 \mathrm{~nm}$ in size, they comprise of micromolecular materials in which the active ingredients (drug or biologically active material) is dissolved, entrapped or encapsulated or adsorbed or attached [1,2].

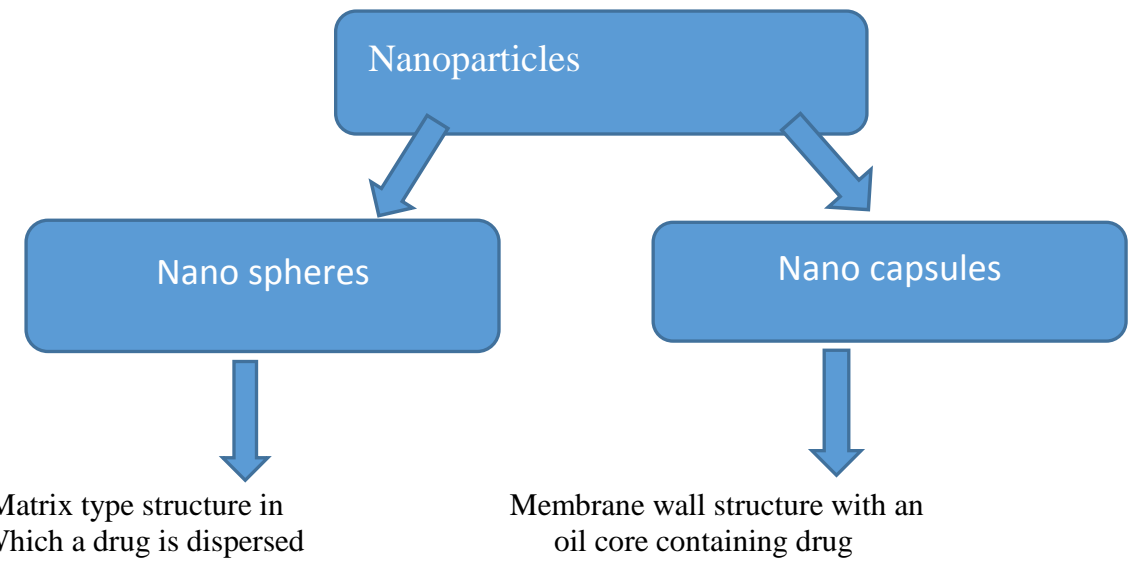




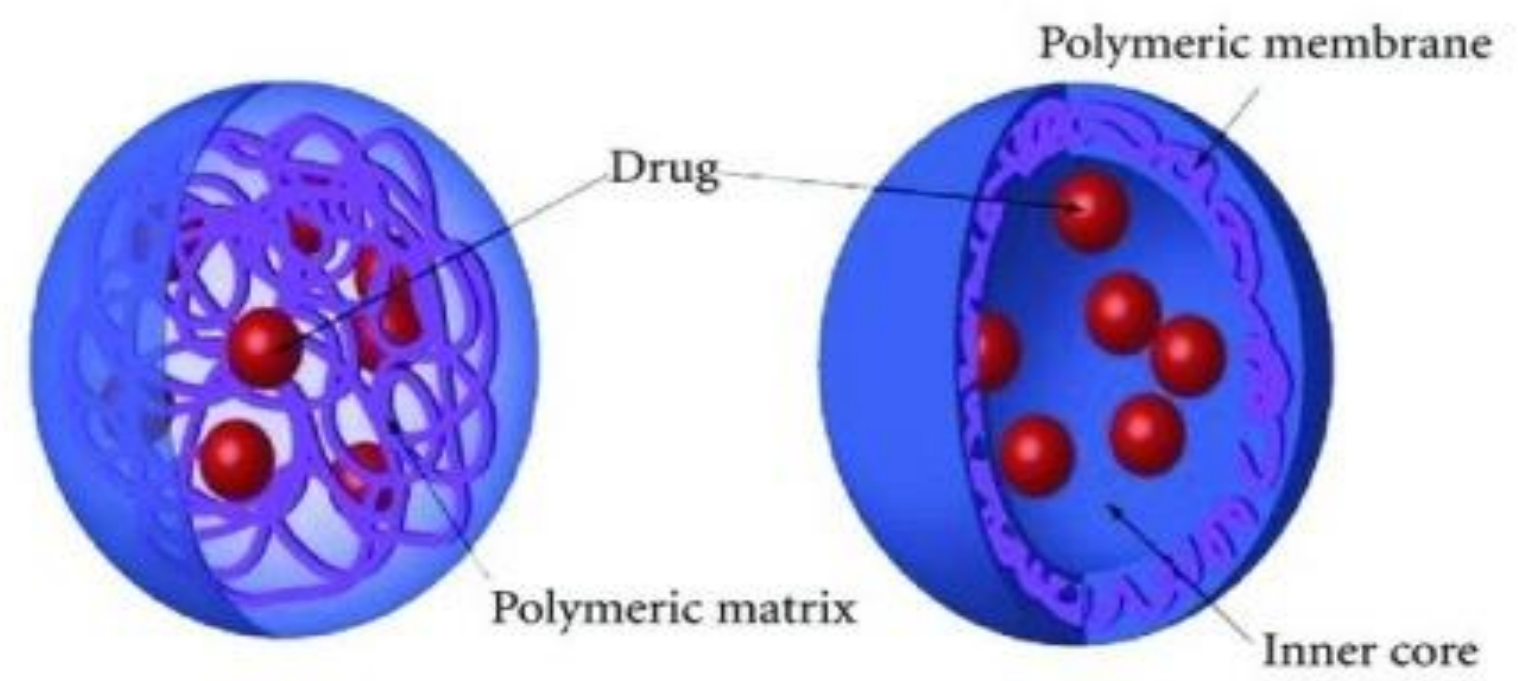

\section{Nanosphere}

\subsection{Advantages $[3,4]$ :}

- Nano particle drug carrier have higher stability.

- Nano particles have higher carrier capacity.

- Nano particles are biodegradable, nontoxic and capable of being stored for longer periods.

- Nano particles can also be used for controlled delivery of drugs.

- Nano particles reduces dosing frequency.

- Nano particles can also administer by parenteral, oral, nasal, or ocular route.

\subsection{Disadvantages:}

- High cost

- $\quad$ Limited drug loading

- Handling of nanoparticles is difficult in liquid and dry forms.

- Polymeric nanoparticles are relatively slowly biodegradable which might cause systemic toxicity.

- Requires skills to manufacture.

- Highly sophisticated technology.

- Difficult to maintain stability of dosage form, E.g., released erythrocytes stored at $4^{\circ} \mathrm{C}$

\subsection{Applications $[5,6]$ :}

- Delivery of anti- cancer drugs.

- Nanoparticles have been found to assemble in tumors after IV administration.

- Reduction in toxicity of anti-cancer drugs as drugs are concentrated mainly in liver and spleen.

- Occular Delivery: nanoparticles with steroids, antiinflammatory agents and bacterial agents for glaucoma in order to improve retention of drug.

- Useful in treatment of hepatic metastases
- Nanoparticles showed an interesting for selective transport of anti-viral agents

EG: Nanoparticles loaded with protease inhibitor was shown to be effective in HIV infected human.

\section{Materials:}

DRUG: Sulfasalazine

POLYMER: BSA

CROSS LINKING AGENT: Glutaraldehyde

SOLVENT: Acetone

\section{Methods:}

\subsection{Method of Preparation of Sulfasalazine Nanoparticles $[7,8]$ :}

Desolvation Technique:

In this method, polymer BSA was weighed and dissolved in $25 \mathrm{ml}$ of distilled water, then the weighed quantity of drug (Sulfasalazine) was added and checked for $\mathrm{pH}$ 7.4. The solvent (Acetone) was added to the above solution dropwise at a rate of $1 \mathrm{ml} / \mathrm{mint}$ in continuous method whereas in intermittent method the solvent was added at a rate of $5 \mathrm{ml} / \mathrm{mint}$ using the designed apparatus under constant stirring at room temperature. The observation of turbidity in the solution indicated the formation of the nanoparticles. For the stabilization of the unstable particles, Glutaraldehyde was added as crosslinking agent for the above solution and the stirring condition was continued for 6hrs. The obtained nanoparticles are Rota evaporated under vacuumed at a temperature of $56^{\circ} \mathrm{c}$ till the solvent is completely evaporated and the obtained nanoparticles are air dried as shown in Figure 1. 


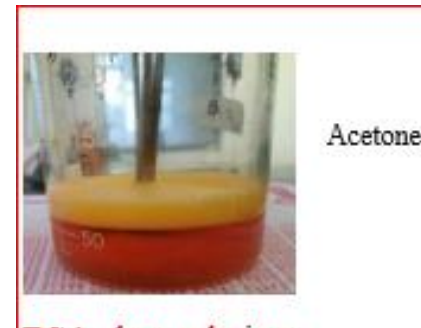

\section{BSA clear solution}

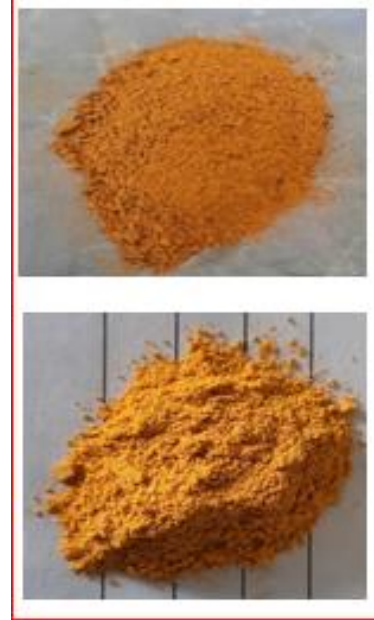

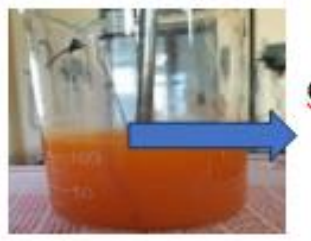

Glutaraldehyde

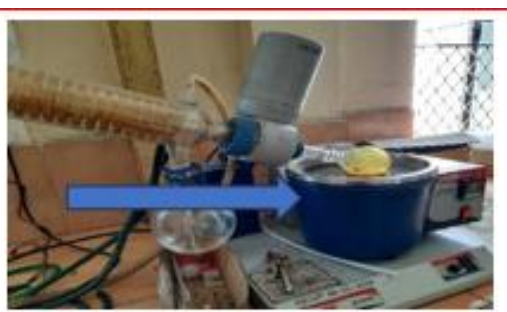

Turbid solution
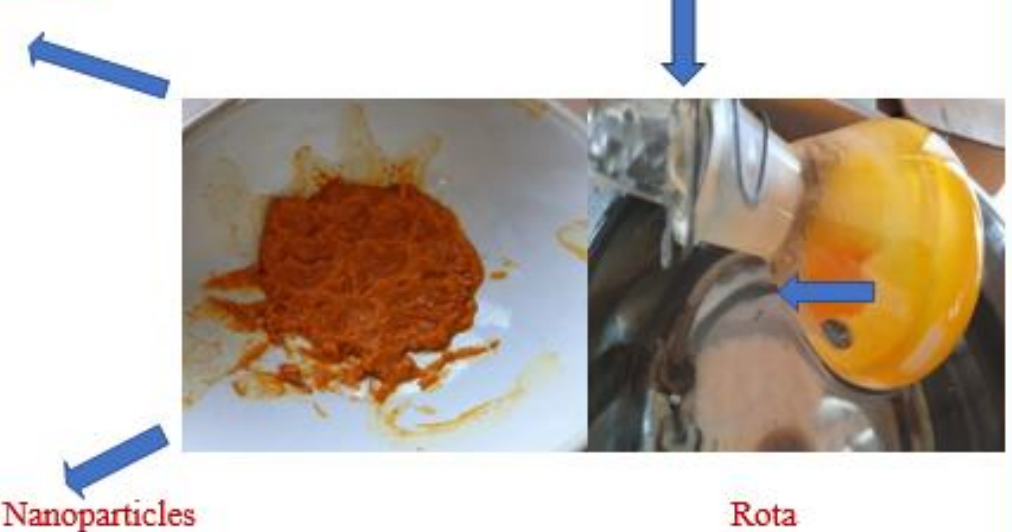

Rota

\section{Under vacuumed}

Figure 1: Experimental work of Sulfasalazine Nanoparticles

\subsection{Evaluation and Characterization of Sulfasalazine Nanoparticles:}

The obtained formulations of sulfasalazine loaded nanoparticles by desolvation technique are evaluated and characterized for the following parameters [9].

\subsubsection{Drug Content $[10,11]$ :}

Free drug of the formulations was first resoluted in the supernatant by selecting a solvent in which only the free drug gets dissolved and not the other ingredients. To determine the drug content, $50 \mathrm{mg}$ drug equivalent to formulation was weighed accurately and transferred into $100 \mathrm{ml}$ beaker containing $50 \mathrm{ml}$ dimethyl sulfoxide (DMSO). The solution was stirred by using magnetic stirrer by keeping at 700rpm for $3 \mathrm{hrs}$. The resultant solution was filtered and the amount of the drug in the filtrate was estimated after suitable dilution by UV spectrophotometer at 359 $\mathrm{nm}$. The drug content of nanoparticles was calculated according to the equation (1).

Amount of drug present in the sample

Total amount of drug loaded initially

\subsubsection{Entrapment Efficiency [12,13]:}

For determination of entrapment efficiency, the amount of drug present in the clear supernatant after centrifugation was determined by UV spectrophotometer at $359 \mathrm{~nm}$. In this method the nanoparticles $20 \mathrm{mg}$ were dispersed in centrifuge tubes which consist of $20 \mathrm{ml}$ of buffer solution ( $\mathrm{pH}-7.4$ ). These tubes are centrifuged at $15000 \mathrm{rpm}$ for $60 \mathrm{mints}$. The $\% \mathrm{EE}$ is calculated by using the equation (2). supernatant

Total drug content - free drug in

$$
\begin{aligned}
& \% \text { Entrapment efficiency }= \\
& \times 100 \ldots \ldots(2)
\end{aligned}
$$

Total drug content

\subsubsection{Average Particle Size and Zeta Potential:}

The average particle size of the optimized formulations was determined by photo correlation spectroscopy with a particle size analyzer equipped with a SOP software. Samples were prepared by dispersing the suitable amount of nanoparticles in $5 \mathrm{ml}$ of distilled water and ultrasonicated for $1 \mathrm{hr}$. The surface charge or zeta potential was determined by measuring the velocity of the particles suspended in a liquid medium under an applied electric field, zeta potential analyzer is used [14].

\subsubsection{Invitro Drug Release Studies:}

A known amount of nanoparticles was transferred to a conical flask and $50 \mathrm{ml}$ of the phosphate buffer $\mathrm{pH}-7.4$ was added to the flask. Experiment is conducted in an orbital shaker in which temperature and rotation were adjusted to $37^{\circ} \mathrm{c}$ and $100 \mathrm{rpm}$, respectively. At definite time interval of $1 \mathrm{hr}, 1 \mathrm{ml}$ of the sample was withdrawn and replaced with equivalent volume of dissolution medium to maintain the sink conditions. The amount of drug release from the nanoparticles was then analyzed by using UV spectroscopy [15].

\subsubsection{Stability Studies:}

The stability of BSA loaded sulfasalazine nanoparticles was evaluated in terms of its drug content and entrapment efficiency. Samples are taken 
in different bottles and are stored at three different conditions like refrigeration temperature $\left(4^{\circ} \mathrm{c}\right)$, at room temperature $\left(25^{\circ} \mathrm{c}\right)$ and at $>40^{\circ} \mathrm{c}$ for a period of 3 months and then the samples were analyzed for drug content by UV spectroscopy at $359 \mathrm{~nm}$. The results were compared with actual results of before storage [16,17].

\section{Results:}

The obtained formulations were characterized and evaluated for the above-mentioned parameters and the results are discussed as follows:

\subsection{Evaluation and Characterization of Sulfasalazine}

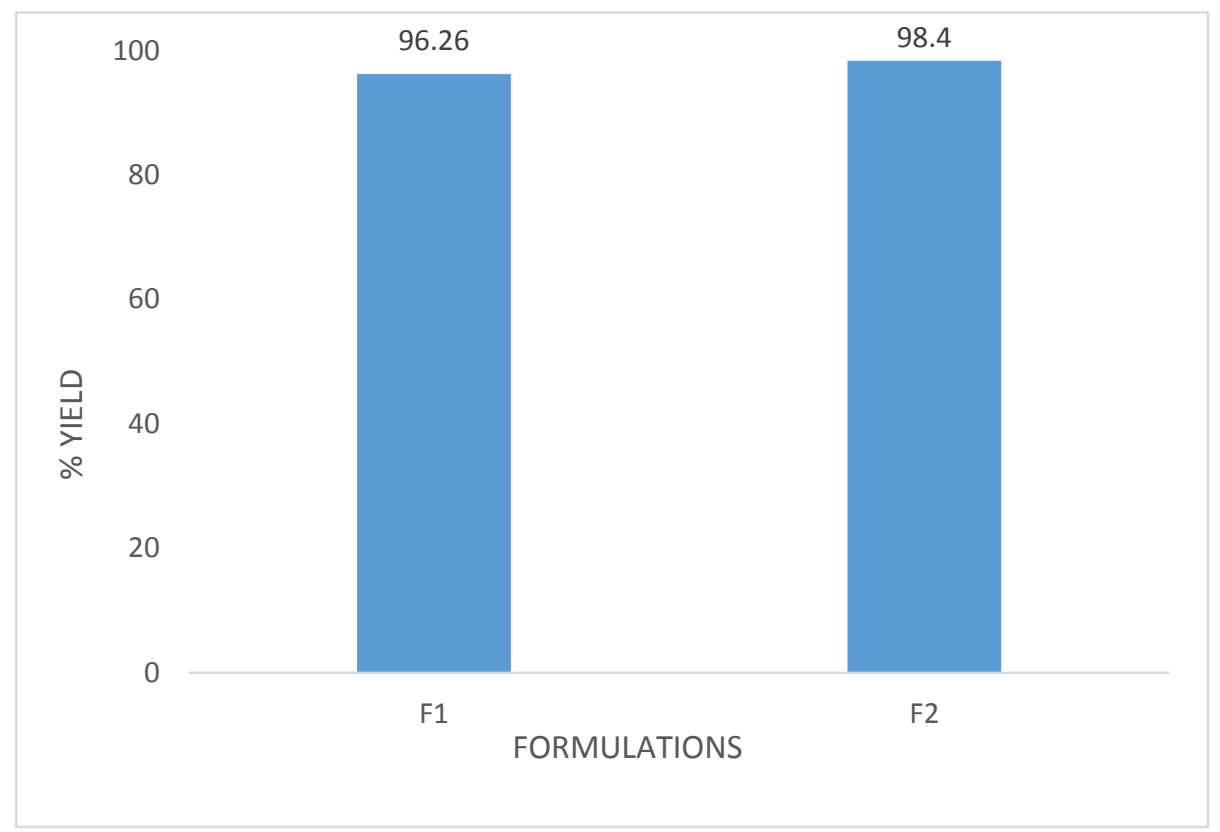

\section{Loaded Nanoparticles by Desolvation Technique:}

Sulfasalazine loaded nanoparticles were prepared by using BSA as polymer by 2 different methods i.e., continuous method and intermittent method. The obtained results for $\%$ yield, drug content and drug release were discussed below.

\section{$\%$ Yeild:}

The percentage yields for both the formulations of sulfasalazine nanoparticles were obtained as $96.26 \%$ for intermittent method (F1) and $98.4 \%$ for continuous method (F2). They are showed in figure 2 .

\section{Figure 2: Comparison of \% Yeld between Intermittent (F1) and Continuous (F2) Methods.}

\section{Drug Content:}

The drug content of both the formulations were compared. They were found to be $95.08 \%$ for F1 (intermittent method) and $97.27 \%$ for F 2 (continuous method) as shown in figure 3.

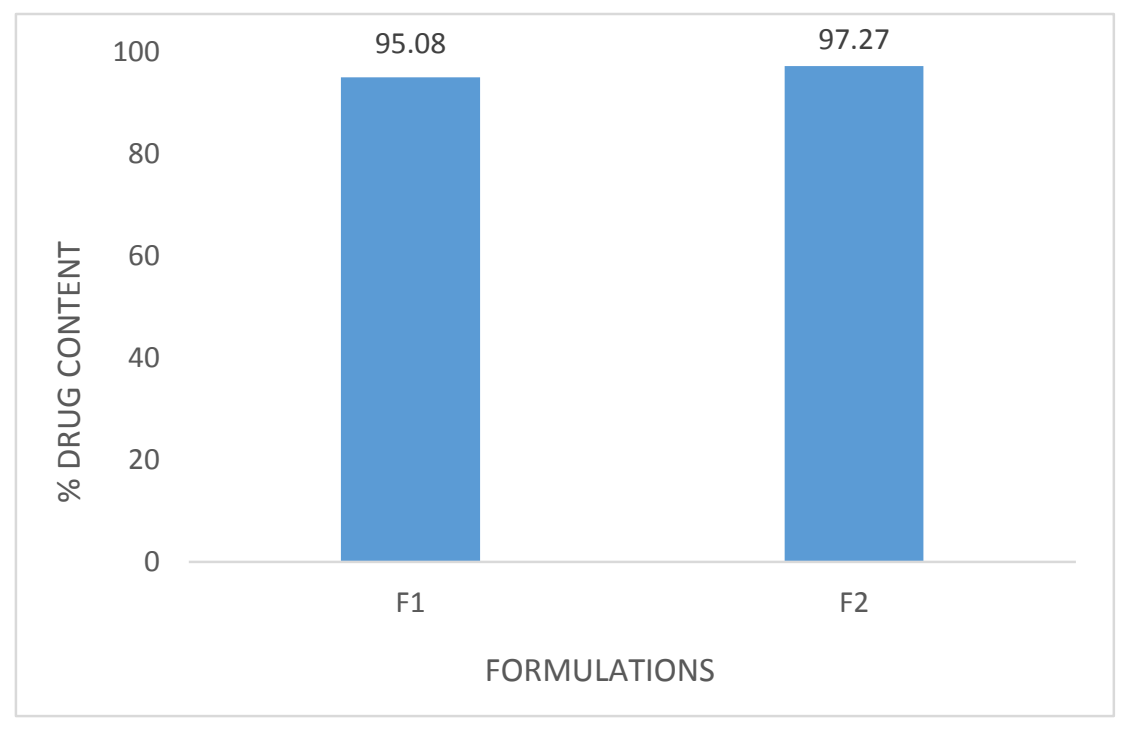




\section{Invitro Drug Release:}

The drug release of both the formulations were compared. They were found to be $40.73 \%$ for F1 (intermittent method) and $38.05 \%$ for F2 (Continuous method) within a span of 6hrs was shown in figure 4.

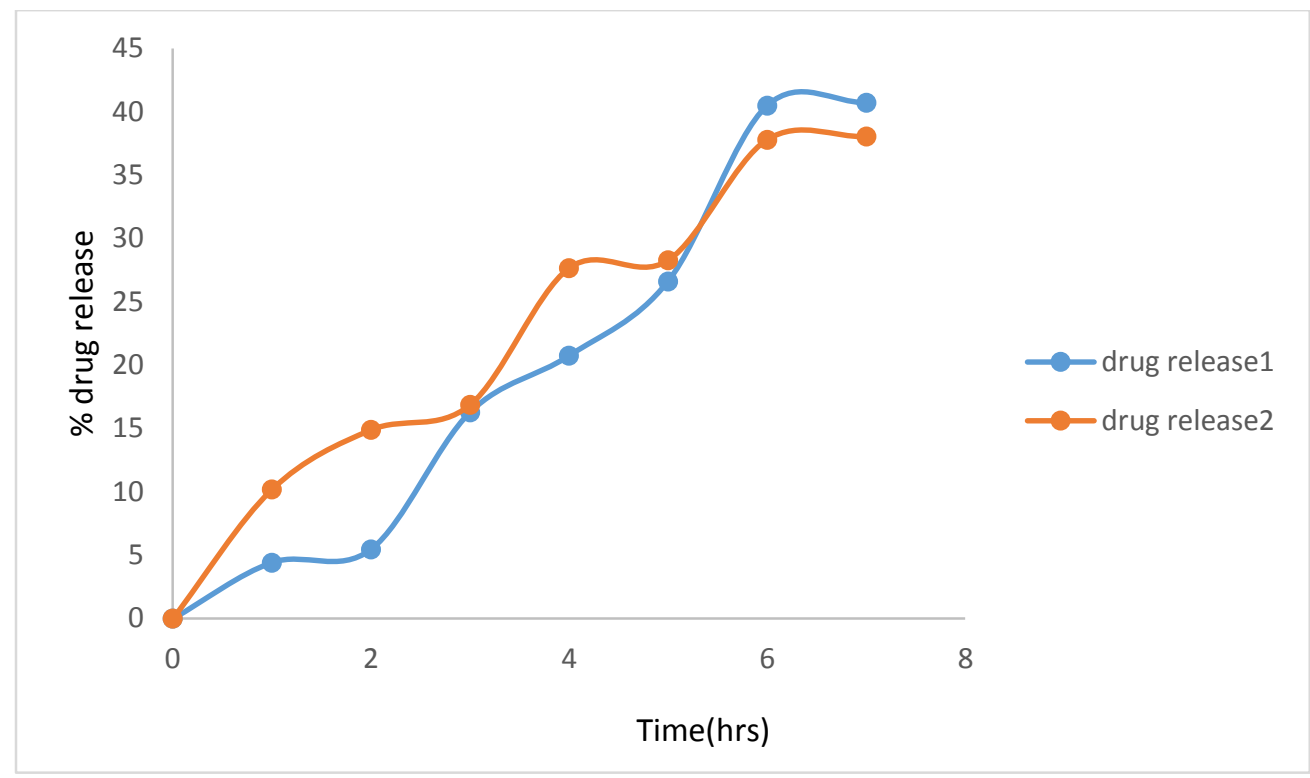

Figure 4: Comparison of \% drug release between F1 and F2 formulations

\section{Discussion:}

Sulfasalazine is a drug known as a disease modifying anti-rheumatic drug (DMARD). It is also used to treat psoriatic arthritis, arthritis associated with inflammatory bowel disease (sometimes known as IBD) and juvenile idiopathic arthritis. Sulfasalazine is not recommended for those who have allergy to salicylates.

On prolong usage of sulfasalazine may cause Dyspepsia, nausea, abdominal discomfort, rashes and macrocytosis.In order to reduce the dosing frequency and adverse effects of the drug, novel approach has been implementedin this study to prepare sulfasalazine loaded nanoparticles.

In this present study sulfasalazine nanoparticles were prepared by desolvation technique by using BSA as polymer, Acetone as solvent and Glutaraldehyde as crosslinking agent. This study was performed to determine the effect of parameters such as drug content, \% yield and invitro drug release was determined.

Among the prepared formulations it was observed that F1 formulation i.e., continuous method of preparation technique was considered as best technique for nanoparticles preparation because it showed highest drug content $(97.27 \%)$ and highest \% yield $(98.4 \%)$ and drug release of $38 \%$ within $6 \mathrm{hrs}$.

\section{Conclusion:}

Sulfasalazine is an anti-rheumatic agent and contains anti-inflammatory properties. In the present study, sulfasalazine loaded with BSA nanoparticles were prepared by using desolvation technique. Nanoparticles are formulated by using continuous and intermittent method and the obtained formulations were evaluated for drug content and drug release. The formulation F2 showed the best results when compared to intermittent method. Based on the above results it was concluded that desolvation technique was considered as the desirable and ideal ideal technique for the preparation of sulfasalazine nanoparticles.

\section{Abbreviations:}

$\% \quad$ Percentage

EE Entrapment efficiency

DC Drug content

UV Ultra violet spectroscopy

$\mathrm{Hr} \quad$ Hour

Min Minutes

RPM Revolutions per minute

$\mathrm{Mg} \quad$ Milligram

BSA Bovine Serum Albumin

$\mathrm{Mg} / \mathrm{ml} \quad$ Milligrams per millilitre

$\mu \mathrm{g} / \mathrm{ml} \quad$ Micrograms per millilitre

DMSO Dimethyl Sulfoxide

$\mathrm{nm} \quad$ Nano meters

DMARD Disease Modifying Anti-Rheumatic Drug

\section{References}

1. Jain N. K, Controlled and novel Drug Delivery, $1^{\text {st }}$ edition (2001), CBS Publication; 292-301.

2. Khar RK, Vyas SP. (2002) Nanoparticles in targeted and controlled drug delivery novel carrier systems. New Delhi: CBS publishers and distributors. 331-385.

3. Thandapani Gomathi, Sudha P.N, Bronze-Uhle ES, Costa BC, Ximenes VF, Lisboa-Filho PN. (2017) Synthetic nanoparticles of bovine serum albumin with entrapped salicylic acid. Nanotechnol Sci Appl; 10:11-21. 
4. Migneault I, Dartiguenave C, Bertrand MJ, Waldron KC. (2004) Glutaraldehyde: behaviour in aqueous solution, reaction with proteins, and application to enzyme crosslinking. BioTechniques; 37:790-802.

5. Rohiwal S, Satvekar R, Tiwari A, Raut A, Kumbhar S, Pawar S. (2015) Investigating the influence of effective parameters on molecular characteristics of bovine serum albumin nanoparticles. Appl Surf Sci; 334:157-64.

6. Paik SYR, Nguyen HH, Ryu J, Che JH, Kang TS, Lee JK, et al. (2013) Robust size control of bovine serum albumin (BSA) nanoparticles by intermittent addition of a desolvating agent and the particle formation mechanism. Food Chem; 141:695-701.

7. Weber C, Coester C, Kreuter J, Langer K. (2000) Desolvation process and surface characteristics of protein nanoparticles. Int $\mathrm{J}$ Pharm; 194:91-102.

8. Yerokun T, Winfield LL. (2015) Celecoxib and LLW-3-6 Reduce Survival of Human Glioma Cells Independently and Synergistically with Sulfasalazine. Anticancer Res.35 (12):641924.

9. Rahimnejad M, Najafpour G, Bakeri G. (2012) Investigation and modelling effective parameters influencing the size of BSA protein nanoparticles as colloidal carrier. Colloids Surf A. 412:96100.

10. Krishna Sailaja A, Amareshwar P. (2012) Preparation of alginate nanoparticles bydesolvation technique using acetone as desolvating agent. Asian journal of pharmaceutical and Clinical Research. 5:2. ISSN-0974-2441

11. Krishna Sailaja A, Amareshwar P, Jayaprakash D. (2013) Preparation of Aspirin loaded BSA nanoparticles by Desolvation techniqueusing sodiumsulphateas desolvating agent. International Journal of Pharmacy and Integrated Life Sciences.6 (13):69-75. 10.

12. Weber C, Coester C, Kreuter J, Langer K. (2000) Desolvation process and surface characterisation of protein nanoparticles. International Journal of Pharmaceutics. 194(1):91-102.

13. Krishna Sailaja A, Chandavat Vineela. (2014) Preparation and characterization of mefenamic acid loaded bovine serum albumin nanoparticles by desolvation technique using acetone as desolvating agent. Der Pharmacia Lettre.6 (6):207-226.

14. Hoang Hai Nguyen, Sanghoon ko. Preparation of size-controlled BSA nanoparticles by intermittent addition of desolvating agent. IFMBE proceedings book series (IFMBE, volume 27).

15. Abbaraju Krishna Sailaja. (2013) Preparation of BSA nanoparticles by desolvation technique using ethyl alcohol as desolvating agent. Journal of Biotechnology and Biomaterials 02(06), 23-25.

16. Krishna Sailaja A, Vutpala Sreelola. (2016) Preparation and characterization of Diltiazem HCL loaded Bovine Serum Albumin nanoparticles by desolvation technique. volume 4 , issue 4 . 